\title{
Estudio y evaluación del diseño de las actividades de aprendizaje de los MOOC de la Colección de Aprendizajes Clave
}

\section{Study and evaluation of the design of the learning activities of the MOOC Coleccion de Aprendizajes Clave}

\author{
Roxana Patricia León González \\ Universidad Autónoma de Baja California. Ensenada, México \\ roxana.leon@uabc.edu.mx \\ Graciela Cordero Arroyo \\ Universidad Autónoma de Baja California. Ensenada, México \\ gcordero@uabc.edu.mx \\ Edna Luna Serrano \\ Universidad Autónoma de Baja California. Ensenada, México \\ eluna@uabc.edu.mx
}

\begin{abstract}
Resumen
Este artículo presenta una evaluación del nivel taxonómico de las actividades de aprendizaje de los MOOC de la Colección de Aprendizajes Clave diseñado para actualizar a profesores de educación primaria de México en enfoques y contenidos del nuevo plan de estudios. Con tal fin se realizó un mapeo de la estructura general de los MOOC. Se definió una tipología de segmentos de actividad (TSA) para realizar un análisis cuantitativo de contenido y clasificar las actividades de aprendizaje de acuerdo a la taxonomía de Anderson y Krathwohl (2001). Esta tipología se valoró por un comité de expertos y se aplicó al conjunto de actividades de aprendizaje identificadas. Se encontró que la mayoría de las actividades se distribuyen en dos de los seis niveles de la taxonomía, el 36\% se relacionan con la comprensión y el $20 \%$ con el análisis. Asimismo, cuatro de los segmentos de actividad corresponden a actividades auténticas que favorecen la transferencia del aprendizaje, lo cual representa el $20 \%$ de las actividades. Se concluye que la TSA es útil tanto para diseñadores como para evaluadores a fin de valorar las demandas cognitivas de un curso masivo y abierto para el logro de sus objetivos.
\end{abstract}

Palabras clave: actividades de aprendizaje, formación de profesores, MOOC, taxonomía, transferencia de la formación.

\begin{abstract}
:
This article presents an evaluation of the taxonomic level of the learning activities of the MOOCs Colección de Aprendizajes Clave design to update elementary school teachers from Mexico in the approach and contents of the new curriculum. To achieve it, a mapping of the general structure of the MOOCs was executed. A typology of segments of an activity (TSA) was defined to perform a quantitative analysis of the content in order to classify the learning activities according to the Anderson and Krathwohl taxonomy (2001). This typology was evaluated by an expert committee and was applied to the whole learning activities identified. It was found that the majority of the activities were distributed in two from the six levels of the taxonomy, $36 \%$ is related to the comprehension and $20 \%$ to analysis.
\end{abstract}


The TSA is useful the designers as well for the evaluators to assess the cognitive demands of an open and massive course for the achievement of the objectives.

Keywords: learning activities, teacher training, MOOCs, taxonomy, transfer of training.

\section{Introducción}

Las tecnologías de la información y la comunicación (TIC) se han integrado a los diferentes ámbitos de la vida diaria, lo cual también ha permeado en la educación, al grado de que cada país ha adoptado reformas para garantizar su adopción y uso en este campo (Del Prete y Cabero, 2020), lo cual ha transformado la forma en que se aprende y se enseña en la actualidad (Vázquez-Cano et al., 2021). Los ambientes de aprendizaje se han diversificado con la incorporación de las TIC que han favorecido el tránsito de la modalidad presencial a la modalidad a distancia (Rivera et al., 2016). Las primeras experiencias de educación a distancia, a través de correspondencia, se dieron en el siglo XIX en el Reino Unido. A finales del siglo XX, gracias a la expansión del uso de la computadora y el Internet se perfilaron las aulas virtuales (Haber, 2014). La utilización de los ambientes virtuales de aprendizaje ha permeado la educación formal y son una alternativa importante para la actualización de recursos humanos (Domínguez y Morcillo, 2016).

En la última década, los Cursos en Línea Masivos y Abiertos (Massive Open Online Courses, MOOC por sus siglas en inglés) se han afianzado como una oferta formativa, que ha generado interés entre la comunidad científica y ha sido objeto de variadas investigaciones (Ruiz-Palmero et al., 2021).Estos dispositivos se incorporan al proceso de aprendizaje en un momento donde los estudiantes y formadores tienen que alcanzar un nivel de competencia teórica y práctica sobre el proceso de enseñanzaaprendizaje en red (Vázquez-Cano et al., 2021). Como menciona Cabero et al. (2014), existen posiciones encontradas respecto al valor de los MOOC, la idea de que representan una tecnología que permite que la educación de calidad llegue a más personas, a través de cursos diseñados por universidades de prestigio a los cuales es posible acceder sin importar la ubicación geográfica, la clase social o la formación previa, contrasta con la concepción de que no representan una innovación en términos educativos y es un modelo de negocios para las universidades. A pesar de las críticas, los MOOC parecen encontrar un espacio de continuidad en la educación (Bozkurt et al., 2016).

Algunos elementos básicos de la definición inicial de MOOC se han modificado en la evolución de esta tendencia. Uno de ellos ha sido el énfasis que ha ganado la masividad, sobre el carácter abierto que en sus inicios era el foco de atención. Otro aspecto que se ha modificado es la visión inicial de que transformarían la educación superior, por una concepción más modesta donde se presentan como una opción más adecuada para la capacitación laboral, lo cual parece guiar el futuro de los MOOC (Haber, 2014). Alemán et al. (2015) mencionan que se requiere efectuar investigaciones a profundidad "para establecer si los MOOC representan oportunidades reales para el aprendizaje en escenarios informales, o si son simplemente intentos de formalizar lo informal" (p. 106).

El diseño instruccional de un MOOC es de especial relevancia. El componente del diseño es el medio por el cual se pueden promover oportunidades óptimas de 
aprendizaje. El formador tiene la tarea de transformar los contenidos en una experiencia deliberada que permita a sus estudiantes la construcción de conocimientos (Maina, 2020), por lo que se considera el componente que tiene mayor influencia sobre el aprendizaje (Zapata-Ros, 2015). En el caso de los MOOC, el diseño es la etapa en la que el formador distribuye los contenidos y las actividades para asegurar el aprendizaje sin estar presente en su gestión; dada su importancia, se han planteado rutas de investigación para indagar cómo el diseño de un MOOC favorece los procesos de aprendizaje de los participantes (Rivera et al., 2016).

Los MOOC se han convertido en una alternativa para la formación continua del profesorado de educación primaria alrededor del mundo. Su carácter autogestionado parece ser un factor favorable para su empleo como estrategia de formación docente. Curtis (2018) menciona que los programas de formación continua en línea deben permitir a los profesores avanzar a su propio ritmo, y no imponer tiempos para que todos los participantes avancen en la misma dirección. Un MOOC brinda la posibilidad de que cada profesor avance según sus necesidades, puesto que no hay fechas definidas ni tiempos preestablecidos para realizar las actividades de aprendizaje. Esto ha provocado interés entre la comunidad académica en diferentes países y se han realizado investigaciones enfocadas al análisis de las experiencias de formación continua de docentes a través de MOOC, por ejemplo, Gynther (2016) en Dinamarca; Oyo et al. (2017) en Uganda; Castaño-Muñoz et al. (2018) en España; King et al. (2018) en Timor Oriental; Mabuan (2018) en Filipinas; Wambugu (2018) en Kenia; y Napier et al. (2020) en Estados Unidos, por mencionar algunos.

Autores como Castaño-Muñoz et al. (2018), Mabuan (2018) y Oyo et al. (2017) encontraron que los cursos estudiados fueron valorados de manera satisfactoria por los maestros participantes, y que representan una oportunidad valiosa para el logro de aprendizajes. No obstante, algunos autores reportaron limitaciones asociadas a los procesos o a los resultados de la formación, como fueron dificultades de acceso a Internet, autopercepción de incompetencia en el manejo de las TIC por parte de algunos profesores (Oyo et al., 2017), limitaciones de tiempo para realizar las actividades y falta de evaluaciones formativas, entre otras (King et al., 2018).

México no ha sido la excepción al adoptar los MOOC como estrategia de formación continua del profesorado. Con el propósito de actualizar a los maestros de educación básica en las características específicas del Plan y programas de estudio para educación básica 2017 denominado Aprendizajes Clave para la Educación Integral (Diario oficial de la Federación [DOF], 2017), la Secretaría de Educación Pública (SEP) ofertó, por primera vez, los MOOC Colección de Aprendizajes Clave (en adelante Colección de AC) a los docentes y directivos de Educación Inicial, Preescolar, Primaria y Secundaria. La oferta de actualización Colección de AC se conformó por 19 cursos en formato MOOC, de los cuales seis corresponden al nivel Primaria (SEP, 2018a). La participación en dicha oferta no fue obligatoria, no obstante, se consideró una responsabilidad y compromiso del docente acreditar el curso correspondiente a su función.

Esta colección resalta por el alcance logrado en términos de cobertura. La población objetivo de este programa de actualización fue de alrededor de 1,200,000 docentes de educación básica, de los cuales 570,000 corresponden al nivel de Primaria (SEP, 2018b) con una edad media de 39 años (Instituto Nacional para la Evaluación de

Estudio y evaluación del diseño de las actividades de aprendizaje de los MOOC de la Colección de Aprendizajes Clave. R. León, G. Cordero y E. Luna. 
la Educación [INEE], 2015). En las primeras seis semanas de su publicación se inscribieron al curso 675,000 docentes (Moreno, 2018). La Dirección General de Formación Continua, Actualización y Desarrollo Profesional de Maestros de Educación Básica (DGFC) registró a lo largo de 2018 a 914,030 participantes, de los cuales 454,670 finalizaron el curso (DGFC, 2019). La Colección de AC probablemente constituya la estrategia de formación continua con mayor alcance, a la fecha, propuesta por el gobierno mexicano para actualizar a más de un millón de maestros de educación básica en todo el territorio nacional. La colección se diseñó para cursarse de forma autogestionada e integró una variedad de recursos por medio de los cuales se distribuye el contenido (videos, archivos en formato PDF, Word, PowerPoint, etc.). Los maestros ubicados en comunidades sin acceso a Internet podían descargar el curso y materiales de apoyo en formato PDF para realizar las actividades de manera offline y subir posteriormente los productos a la plataforma. La comunicación se promovía a través de foros de discusión asincrónicos.

Hasta el momento, lo que se sabe de los resultados de la implementación de estos MOOC es lo publicado por la propia SEP. En los Resultados de la Oferta de Formación Continua 2018 se concentraron las respuestas de 29,300 participantes que realizaron la encuesta de satisfacción al finalizar el MOOC. En términos generales, el curso fue valorado de manera satisfactoria, en relación con su estructura y organización, la relevancia del contenido y la claridad de los propósitos formulados para el curso. La mayoría de los participantes señaló también que el curso fue de utilidad en la mejora de su competencia pedagógica (SEP, 2018b).

Kirkpatrick y Kirkpatrick (2016) mencionan que, si bien las encuestas de satisfacción son útiles para obtener retroalimentación sobre el proceso de formación, la información que proporcionan puede ser limitada, además de que la opinión sobre la formación recibida puede cambiar una vez que los participantes intenten aplicar lo aprendido. Por su parte, Guskey (2002) indicó que se requiere de una evaluación en distintos niveles para promover la mejora de los programas de formación, ya que las encuestas de satisfacción se ubican en el nivel inferior de la evaluación. Si se desea tener un conocimiento más profundo sobre los resultados de la formación, estos autores recomiendan adentrarse en el estudio de los otros niveles, como: la identificación de los logros de aprendizaje, el apoyo y cambio institucional, la transferencia de la formación, y el impacto en el puesto de trabajo.

Ante el gran alcance de la Colección de AC para el profesorado mexicano en términos de la cobertura, se consideró objeto de interés generar evidencia empírica que permita la valoración del diseño de sus actividades de aprendizaje. Dado su propósito específico de actualización de enfoques y contenidos del nuevo plan de estudios, se planteó indagar las demandas cognitivas de sus actividades de aprendizaje a fin de saber si permiten que los docentes transfieran o apliquen lo aprendido en su práctica de aula.

Con el fin de aproximarse a los procesos de aprendizaje y transferencia al aula, se eligió la taxonomía de Anderson y Krathwohl (2001) como el modelo conceptual para el análisis, dado que permite hacer explícitas las demandas cognitivas asociadas a las actividades de aprendizaje (Tee et al., 2010). Con base en la clasificación de esta taxonomía, Mayer (2002) refiere que el nivel Recordar se orienta hacia la retención del conocimiento, mientras que los niveles taxonómicos Comprender, Aplicar, Analizar, Evaluar y Crear promueven la aplicación de lo aprendido. Es así como la Taxonomía

Estudio y evaluación del diseño de las actividades de aprendizaje de los MOOC de la Colección de Aprendizajes Clave. R. León, G. Cordero y E. Luna. 
provee una estructura que facilita la valoración de las actividades, además de establecer una distinción entre aquellos que promueven la retención y la transferencia del aprendizaje.

El objetivo del artículo es evaluar el diseño de las actividades de aprendizaje propuestas en los seis MOOC de la Colección de AC para educación primaria, mediante la identificación del nivel taxonómico del proceso cognitivo implicado en el diseño de las actividades, para hacer explícitas las demandas cognitivas implicadas en la formación, y su factibilidad para transferirlo al aula del docente.

\section{Método}

Este estudio es de tipo descriptivo. El proceso de diseño del instrumento para la evaluación del nivel taxonómico de las actividades de aprendizaje de la Colección de AC se basó en la aplicación de la técnica de análisis cuantitativo de contenido a partir de la taxonomía revisada de Anderson y Krathwohl (2001. Duverger (1983) se refiere al análisis cuantitativo de contenido como una forma particular de semántica cuantitativa, donde el objetivo es extraer lo esencial de un conjunto considerable de datos y se busca eliminar la subjetividad al lograr una interpretación independiente de la personalidad del intérprete. Asimismo, se empleo el juicio de expertos en la búsqueda del consenso intersubjetivo de la propuesta de clasificación de las actividades de aprendizaje (Jornet et al., 2011).

\section{Participantes}

Para el logro del propósito del estudio se contó con dos tipos de participantes: expertos que integraron un comité inicial de trabajo y codificadores para el análisis cuantitativo de contenido.

Se convocó a un comité de expertos con la finalidad de valorar una tipología de segmentos de actividad (TSA) elaborada para el análisis cuantitativo de contenido de las actividades de aprendizaje. El comité estuvo conformado por cinco profesionistas con diferentes perfiles (ver Tabla 1), todos del campo de la educación. 
RED. Revista de Educación a Distancia. Núm. 67, Vol. 21. Artíc. 9, 30-Sep -2021

DOI: http://dx.doi.org/10.6018/red.478311

\section{Tabla 1}

Características de los participantes del comité de expertos

\begin{tabular}{|c|c|c|c|c|}
\hline $\begin{array}{l}\text { Ocupación } \\
\text { actual }\end{array}$ & Institución & $\begin{array}{l}\text { Grado de } \\
\text { estudio }\end{array}$ & Experiencia en docencia & $\begin{array}{l}\text { Experiencia en } \\
\text { investigación }\end{array}$ \\
\hline $\begin{array}{l}\text { Supervisora } \\
\text { escolar }\end{array}$ & $\begin{array}{l}\text { Secretaría de } \\
\text { Educación de Baja } \\
\text { California }\end{array}$ & Maestría & $\begin{array}{l}19 \text { años como docente en } \\
\text { primaria } \\
10 \text { años como docente en } \\
\text { escuela normal } \\
4 \text { años como docente en la } \\
\text { Universidad Pedagógica } \\
\text { Nacional }\end{array}$ & $\begin{array}{l}\text { Elaboración de } \\
\text { proyectos escolares }\end{array}$ \\
\hline $\begin{array}{l}\text { Asesora de } \\
\text { zona escolar }\end{array}$ & $\begin{array}{l}\text { Secretaría de } \\
\text { Educación } \\
\text { de Baja California }\end{array}$ & Maestría & $\begin{array}{l}14 \text { años en educación } \\
\text { primaria }\end{array}$ & $\begin{array}{l}\text { Elaboración de } \\
\text { instrumentos de } \\
\text { evaluación } \\
\text { Participación en la } \\
\text { elaboración de la retícula } \\
2011-2017\end{array}$ \\
\hline Docente & Escuela Normal & Maestría & $\begin{array}{l}10 \text { años como docente en } \\
\text { primaria y secundaria } \\
15 \text { años como docente en } \\
\text { escuela normal }\end{array}$ & $\begin{array}{l}\text { Diseño, desarrollo y } \\
\text { difusión de proyectos de } \\
\text { investigación }\end{array}$ \\
\hline $\begin{array}{l}\text { Estudiante } \\
\text { de doctorado }\end{array}$ & Universidad privada & Maestría & $\begin{array}{l}5 \text { años como docente en } \\
\text { cursos virtuales de maestría } \\
\text { en educación }\end{array}$ & $\begin{array}{l}\text { Investigación educativa } \\
\text { sobre MOOC de } \\
\text { matemáticas }\end{array}$ \\
\hline Investigador & Universidad pública & Doctorado & $\begin{array}{l}\text { En educación superior y } \\
\text { media superior }\end{array}$ & $\begin{array}{l}\text { Desarrollo de proyectos } \\
\text { de investigación }\end{array}$ \\
\hline
\end{tabular}

En el estudio también participaron tres codificadoras, para efectos de reportar el índice de fiabilidad del análisis cuantitativo de contenido. La codificadora 1 (C1) es quien desarrolló la TSA y participó en todo el desarrollo de esta investigación. La codificadora 2 (C2) y la codificadora 3 (C3) fungieron como colaboradoras externas; ambas con formación en el campo de la investigación educativa y experiencia en la realización de análisis de contenido.

\section{Materiales}

A lo largo de las fases del estudio se utilizaron diversos materiales. En la Tabla 2 se presentan de acuerdo con cada fase. 


\section{Tabla 2}

Materiales empleados en las distintas fases de la investigación

\begin{tabular}{ll}
\hline Fases & Materiales \\
\hline 1. Mapeo de la estructura de la & $\begin{array}{l}\text { Plataforma en línea que soporta los MOOC de la Colección de } \\
\text { AC }\end{array}$ \\
& $\begin{array}{l}\text { Transcripción de las actividades de aprendizaje de los MOOC de } \\
\text { la Colección de AC para educación primaria }\end{array}$ \\
$\begin{array}{l}\text { 2. Diseño de la Tipología del } \\
\text { Segmento de Actividades }\end{array}$ & $\begin{array}{l}\text { Taxonomía de Anderson y Krathwohl (2001) } \\
\text { Primera versión de la TSA }\end{array}$ \\
$\begin{array}{l}\text { 3. Valoración de la TSA por comité } \\
\text { de expertos }\end{array}$ & $\begin{array}{l}\text { Primera versión de la TSA } \\
\text { Cuadernillo de trabajo con listas de cotejo para la valoración de la } \\
\text { TSA }\end{array}$ \\
$\begin{array}{l}\text { 4. Identificación del nivel } \\
\text { taxonómico del proceso cognitivo }\end{array}$ & $\begin{array}{l}\text { Guía de codificación } \\
\text { Segunda y tercera versión de la TSA } \\
\text { Paquete Estadístico para Ciencias Sociales (SPSS por sus siglas } \\
\text { en inglés) }\end{array}$ \\
\hline
\end{tabular}

\section{Procedimiento}

El estudio se realizó en cuatro etapas: (1) mapeo de la estructura de la Colección de AC, (2) diseño de la Tipología del Segmento de Actividades (TSA), (3) valoración de la TSA por comité de expertos y (4) identificación del nivel taxonómico del proceso cognitivo.

La primera etapa consistió en el análisis de la estructura de los MOOC de la Colección de AC para profesores de educación primaria. Se exploró la plataforma que los soporta con el propósito de identificar la estructura general de los cursos, las actividades de aprendizaje plasmadas, los recursos y materiales propuestos, etc. Además, en esta etapa se realizó la transcripción de todas las actividades de aprendizaje, se identificaron lecciones repetidas en dos o más grados para, finalmente, hacer una síntesis de la estructura general de la Colección de AC.

En la segunda etapa del estudio se diseñó una TSA para ser empleada como un sistema de categorías para el análisis de contenido cuantitativo. Se tomó como referencia la definición de Duverger (1983) y se retoma lo expresado por Krippendorff (2004) en relación con que es "una técnica de investigación para hacer inferencias replicables y válidas de textos (u otra materia significativa) a los contextos de su uso" (p. 18).

Para el diseño de la TSA se transcribieron las instrucciones de las actividades de aprendizaje de las 20 lecciones que componen la Colección de AC, que en total fueron 80 actividades. A partir de esto, se identificó que las actividades no son uniformes, en relación con el número de acciones que el docente debe realizar, así como los productos solicitados en éstas, es por ello que se consideró necesario definir una unidad de análisis. Para su definición se optó por emplear el concepto de segmento de actividad formativa, que es definido como "un fragmento temporal en el cual la actividad educativa compartida está orientada a conseguir un objetivo por parte del formador que coordina y gestiona la interacción” (Badia y Monereo, 2004, p. 55). 
El segmento de actividad formativa es un período temporal en el que las acciones de los participantes se orientan a conseguir ciertos propósitos, en dicho momento los participantes utilizan el mismo tipo de procedimientos instrumentales y psicológicos. Para esta investigación se considera como segmentos de actividad los fragmentos de instrucción en los que al profesor se le solicita llevar a cabo una o más actividades, orientadas a la realización de un producto en específico, por ejemplo: responder preguntas, encontrar diferencias y semejanzas entre la información presentada, recuperar las ideas centrales, elaborar un esquema, etc. Para delimitar los segmentos de actividad formativa se tomó en cuenta lo señalado por Badia y Monereo (2004), respecto a que se considera un cambio de segmento de actividad formativa cuando las acciones y los instrumentos empleados por los docentes cambian. Es así que, de las 80 actividades transcritas en un principio resultaron 152 segmentos de actividad, a partir de los cuales se diseñó un sistema de categorías basado en los niveles del proceso cognitivo de la Taxonomía de Anderson y Krathwohl (2001) denominado TSA para la realización del análisis cuantitativo de contenido. Con base en ello, se creó un sistema de categorías de orden jerárquico, que se construyó a manera de libro de códigos para el análisis, conformado por: categorías, tipología de segmentos, definición de cada tipo de segmento y un ejemplo en cada caso.

En la tercera etapa se empleó la estrategia de juicio de expertos para aportar evidencias de validez de la TSA propuestas para el análisis de contenido. Escobar-Pérez y Cuervo-Martínez (2008) definen el juicio de expertos como "una opinión informada de personas con trayectoria en el tema, que son reconocidas por otros como expertos cualificados en éste, y que pueden dar información, evidencia, juicios y valoraciones" (p. 29). Por medio del juicio de expertos se buscó que las definiciones de las categorías fueran congruentes con la Taxonomía de Anderson y Krathwohl (2001), así como pertinentes para el análisis cuantitativo de contenido. De igual forma, se intentó que fueran exhaustivas y mutuamente excluyentes, como lo indica López-Aranguren (2000).

El comité de expertos validó, mediante juicios independientes, de las categorías propuestas para el análisis de contenido de las actividades de aprendizaje de la Colección de AC. Para tales efectos, se llevó a cabo una sesión de trabajo, la cual se desarrolló en tres fases:

(1) Valoración de la congruencia entre: (a) el nivel taxonómico del proceso cognitivo, (b) la TSA, y (c) la definición de cada uno de los tipos de segmentos.

(2) Valoración de la pertinencia de los elementos analizados en la fase anterior para clasificar las actividades de aprendizaje.

(3) Valoración de la pertinencia del empleo de la TSA para la clasificación de actividades de aprendizaje de los MOOC de la Colección de AC para profesores de primero de primaria.

Una vez realizados los ajustes a la TSA, con base en las observaciones del comité de expertos, se procedió a la cuarta etapa que consistió en la codificación de las actividades de aprendizaje por medio de la TSA. Se decidió emplear la estrategia de inter-codificación para medir el grado de acuerdo entre codificadores, y de esta forma examinar el funcionamiento de las categorías propuestas para el análisis de contenido. Para determinar el grado de acuerdo entre codificadores se empleó el coeficiente $\alpha$ de Krippendorff (2004). En relación con el índice de confiabilidad esperado, se hacen 
algunas anotaciones. En primer lugar, que no existe un índice de confiabilidad referenciado por alguna norma, sino que depende del criterio del investigador. No obstante, se recomienda que un índice no sea demasiado bajo si se pretende que los resultados puedan ser considerados con seriedad, es por ello que para sacar conclusiones tentativas se recomienda un índice de fiabilidad de entre 0.67 y 0.8 (Krippendorff, 2004). Para realizar las pruebas de fiabilidad se utilizó el paquete estadístico SPSS) versión 25 y se siguió la ruta de análisis descrita por Hayes y Krippendorff (2007).

Se realizaron cuatro rondas de codificación, en las que participaron tres codificadoras. En la primera ronda las codificadoras realizaron una revisión de la TSA, con la cual se hicieron los ajustes finales a las categorías para la codificación.

\section{Resultados}

Se procedió a la integración de los hallazgos una vez alcanzado el índice de acuerdo dentro de lo esperado (0.87), lo cual indica un consenso casi total entre las tres codificadoras. La integración consistió en el análisis de los resultados del estudio, al considerar que los datos obtenidos son confiables para realizar las interpretaciones correspondientes, en donde se calcularon frecuencias y porcentajes de los TSA identificados y de los niveles taxonómicos del proceso cognitivo, asimismo, se realizaron representaciones gráficas de los datos.

\section{Mapeo de la estructura de la Colección de AC}

A través de la exploración de la plataforma virtual se identificaron algunas características generales de los MOOC que conforman la oferta de la Colección de AC, como son el diseño de la plataforma, los materiales y recursos para los usuarios, la batería de lecciones y la estructura temática de los cursos. Enseguida se describen dichos elementos.

Diseño de la plataforma. En la página principal se despliega el menú al centro con todos los cursos de la colección, los cuales son accesibles a través de la opción de auto-registro. En la misma página se presenta un panel de navegación que permite: desplazarse entre los cursos inscritos (por grado y por lección), acceder a los archivos privados, calificaciones, calendario, etc. La sesión expira a los cinco minutos de inactividad.

Materiales y recursos para los usuarios. En el curso se ofrece una variedad de materiales de consulta y recursos interactivos, así como videos con información recuperada del Plan y los programas de estudio 2017, con opiniones de especialistas sobre dichas temáticas, fragmentos de conferencias, etc. Además, incluye archivos descargables en formato PDF, Word y PowerPoint en tanto que no todos los maestros tienen acceso permanente a internet y suelen descargar los materiales para trabajarlos fuera de línea. También se incluye una variedad de recursos interactivos, como son: espacios para añadir productos elaborados y comentarios sobre los mismos, foros de discusión asincrónicos, espacios colaborativos para la creación de textos y pruebas de opción múltiple con retroalimentación instantánea.

Batería de lecciones y la estructura temática de los cursos. Por medio del mapeo de la estructura de la Colección de AC se identificó que algunas de las lecciones se repetían entre grados, e inclusive algunas son idénticas para todos los MOOC. En la 
estructura general se reconoció que cada curso se conforma por diez lecciones, subdivididas por temas y actividades. Es así como, en teoría, la colección para el nivel primaria estaría conformado por 60 unidades. Sin embargo, se identificó que la colección de cursos está constituida sólo por una batería de 20 lecciones únicas y diferentes, es decir, aquellas que se distinguen unas de otras por la organización de las actividades de aprendizaje y las temáticas que se abordan. A partir de esta batería de lecciones se estructuran los seis cursos para educación primaria (ver Figura 1).

\section{Figura 1}

Batería de lecciones que conforman los MOOC de la Colección de AC

\begin{tabular}{|c|c|c|c|c|c|}
\hline \multicolumn{6}{|c|}{ Cursos de la Colección de Aprendizajes Clave } \\
\hline $1^{\circ}$ & $2^{\circ}$ & $3^{\circ}$ & $4^{\circ}$ & $5^{\circ}$ & $6^{\circ}$ \\
\hline \multicolumn{6}{|c|}{$\begin{array}{l}\text { Aprendizajes clave para la educación integral Plan y Programas de estudio } \\
\text { (1) }\end{array}$} \\
\hline $\begin{array}{l}\text { Dosificación } \\
\text { curricular. } \\
\text { Los } \\
\text { programas de } \\
\text { estudio de } \\
\text { Primer grado } \\
\text { (2) }\end{array}$ & $\begin{array}{l}\text { Dosificación } \\
\text { curricular. Los } \\
\text { programas de } \\
\text { estudio de } \\
\text { Segundo } \\
\text { grado (3) }\end{array}$ & $\begin{array}{l}\text { Dosificación } \\
\text { curricular. Los } \\
\text { programas de } \\
\text { estudio de } \\
\text { Tercer grado } \\
\text { (4) }\end{array}$ & $\begin{array}{c}\text { Dosificación } \\
\text { curricular. } \\
\text { Gradualidad } \\
\text { de } \\
\text { Aprendizajes } \\
\quad \text { (5) }\end{array}$ & $\begin{array}{l}\text { Dosif } \\
\text { Gradual }\end{array}$ & $\begin{array}{l}\text { ricular. } \\
\text { endizajes }\end{array}$ \\
\hline \multicolumn{2}{|c|}{$\begin{array}{l}\text { Lengua Materna. Español: } \\
\text { propósitos, enfoque } \\
\text { pedagógico y evolución } \\
\text { curricular (7) }\end{array}$} & \multicolumn{4}{|c|}{$\begin{array}{l}\text { Lengua Materna. Español: propósitos, enfoque pedagógico y } \\
\text { evolución curricular (8) }\end{array}$} \\
\hline \multicolumn{2}{|c|}{$\begin{array}{l}\text { Lengua Materna. Español: } \\
\text { adquisición de la lectura y la } \\
\text { escritura en la primaria } \\
\text { (9) }\end{array}$} & \multicolumn{4}{|c|}{$\begin{array}{l}\text { Lengua Materna. Español: Orientaciones didácticas y } \\
\text { sugerencias de evaluación (10) }\end{array}$} \\
\hline \multicolumn{2}{|c|}{$\begin{array}{l}\text { Lengua Materna. Español: } \\
\text { orientaciones didácticas y } \\
\text { sugerencias de evaluación para } \\
\text { primer grado de primaria (11) }\end{array}$} & \multicolumn{4}{|c|}{$\begin{array}{l}\text { Matemáticas. Enfoque pedagógico, propósitos y evolución } \\
\text { curricular. } \\
(12)\end{array}$} \\
\hline \multicolumn{2}{|c|}{$\begin{array}{l}\text { Matemáticas. Enfoque } \\
\text { pedagógico, propósitos y } \\
\text { evolución curricular } \\
\text { (12) }\end{array}$} & \multicolumn{4}{|c|}{$\begin{array}{l}\text { Matemáticas. La utilidad de las orientaciones didácticas y las } \\
\text { sugerencias de evaluación. } \\
\text { (13) }\end{array}$} \\
\hline \multicolumn{2}{|c|}{$\begin{array}{l}\text { Matemáticas. La utilidad de las } \\
\text { orientaciones didácticas y las } \\
\text { sugerencias de evaluación } \\
\text { (13) }\end{array}$} & \multicolumn{4}{|c|}{$\begin{array}{l}\text { Ciencias Naturales y Tecnología } \\
\text { (14) }\end{array}$} \\
\hline $\begin{array}{l}\text { Conocimiento } \\
\text { del medio } \\
\text { (15) }\end{array}$ & $\begin{array}{l}\text { Conocimiento } \\
\text { del medio } \\
\text { (16) }\end{array}$ & $\begin{array}{l}\text { Historias, } \\
\text { paisajes y la } \\
\text { convivencia } \\
\text { en mi } \\
\text { localidad } \\
\text { (17) }\end{array}$ & Historia, Ge & $\begin{array}{r}\text { grafia y F } \\
\text { Ética } \\
(18)\end{array}$ & 'ívica y \\
\hline \multicolumn{6}{|c|}{$\begin{array}{c}\text { Desarrollo personal y social } \\
(19)\end{array}$} \\
\hline \multicolumn{6}{|c|}{$\begin{array}{l}\text { Cierre del curso, evaluación y encuestas de satisfacción } \\
(20)\end{array}$} \\
\hline
\end{tabular}

A través del mapeo también se identificó que los cursos de los seis grados comparten una estructura en relación con las temáticas que se abordan (ver Figura 2). En general, los cursos guardan similitud en las unidades de inicio, desarrollo y cierre. Los seis grados inician con dos lecciones referentes a generalidades del programa de 
estudios de Aprendizajes Clave. Posteriormente, se proponen seis lecciones relacionadas con las asignaturas del Campo de Formación Académica, referentes a las asignaturas de Español, Matemáticas, Ciencias Naturales y Tecnología, Conocimiento del Medio, Historia, Geografía y Formación Cívica y Ética. Finalmente, los seis grados cierran con la lección 19, referente al área de Desarrollo Personal y Social, referente al trabajo en Artes, Educación Socioemocional y Educación Física, y la lección 20 de cierre del curso.

\section{Figura 2}

Estructura temática de la Colección de AC

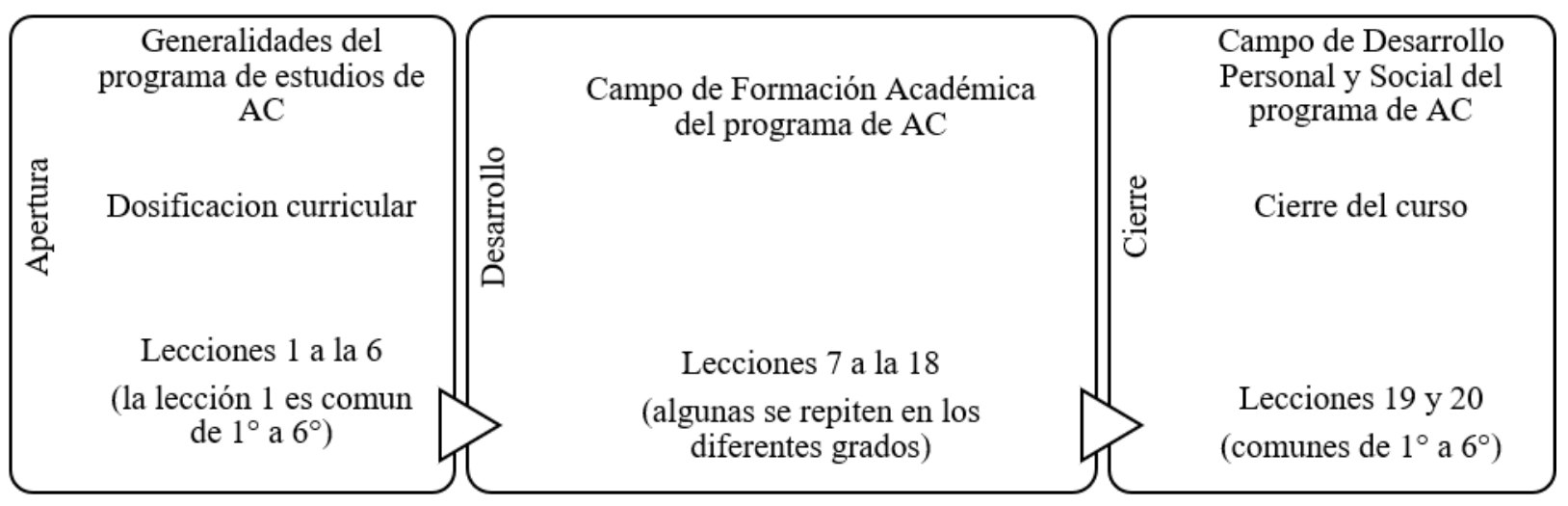

Se observó que en el diseño de los MOOC se dedica un mayor número de lecciones a las asignaturas de Español y Matemáticas en todos los grados. Los cursos para $1^{\circ}$ y $2^{\circ}$ incluyen tres unidades para el estudio de la asignatura de Español, y de $3^{\circ}$ a $6^{\circ}$ se proponen dos sobre la misma asignatura. Mientras que para Matemáticas se plantean dos lecciones por MOOC, las cuales son idénticas para todos los grados.

Asimismo, se identificó que cada una de estas 20 lecciones es independiente de las otras; es decir, no existe relación o continuidad entre las actividades de diferentes lecciones, aun cuando correspondan a la misma temática. La única que guarda relación con otras lecciones es la número 20 , referente al cierre del curso, en la cual se solicita al profesor recuperar información de los conocimientos logrados en las actividades realizadas a lo largo del MOOC.

\section{Diseño de la Tipología de Segmentos de Actividad}

Como se mencionó anteriormente, se diseñó un sistema de categorías denominado TSA basado en la Taxonomía de Anderson y Krathwohl (2001) para efectuar el análisis cuantitativo de contenido, con el objetivo de identificar el nivel taxonómico del proceso cognitivo asociado a las actividades de aprendizaje. Se elaboraron tres versiones de la TSA, para ajustar las distintas versiones se tomaron en consideración las observaciones recuperadas del comité de expertos y los comentarios expresados por las participantes en la primera ronda de la codificación. A partir de los resultados de ambas técnicas se construyó la versión final de la TSA compuesta por 14 categorías (ver Tabla 3). 


\section{Tabla 3}

Versión final de la TSA

\begin{tabular}{|c|c|c|}
\hline Dimensión & $\begin{array}{c}\text { Tipología de segmentos de } \\
\text { actividad }\end{array}$ & Definición \\
\hline \multirow[b]{2}{*}{ Recordar } & $\begin{array}{l}\text { 1. Reconocer información de la } \\
\text { memoria sobre un tema revisado }\end{array}$ & $\begin{array}{l}\text { Demanda al profesor identificar conocimiento } \\
\text { relevante sobre los contenidos revisados en la } \\
\text { lección. }\end{array}$ \\
\hline & $\begin{array}{l}\text { 2. Recuperar información de la } \\
\text { memoria sobre la experiencia } \\
\text { personal }\end{array}$ & $\begin{array}{l}\text { Demanda al profesor recordar aspectos personales } \\
\text { almacenados en la memoria a largo plazo. }\end{array}$ \\
\hline \multirow{3}{*}{ Comprender } & $\begin{array}{l}\text { 3. Ejemplificar acciones empleadas } \\
\text { para la enseñanza }\end{array}$ & $\begin{array}{l}\text { Demanda al profesor identificar acciones para la } \\
\text { enseñanza, desde su experiencia personal. }\end{array}$ \\
\hline & $\begin{array}{l}\text { 4. Abstraer información de un } \\
\text { material o recurso revisado }\end{array}$ & $\begin{array}{l}\text { Demanda al profesor construir una representación } \\
\text { de puntos centrales o importantes de la información } \\
\text { presentada en un material o recurso. }\end{array}$ \\
\hline & 5. Comparar ideas & $\begin{array}{l}\text { Demanda al profesor identificar similitudes o } \\
\text { diferencias entre dos o más fuentes de información. }\end{array}$ \\
\hline Aplicar & $\begin{array}{l}\text { 6. Llevar a cabo una serie de } \\
\text { indicaciones para elaborar una } \\
\text { planeación }\end{array}$ & $\begin{array}{l}\text { Demanda al profesor elaborar una planeación } \\
\text { didáctica guiada por una serie de elementos. }\end{array}$ \\
\hline \multirow{3}{*}{ Analizar } & 7. Organizar conceptos o ideas & $\begin{array}{l}\text { Demanda al profesor identificar conceptos o ideas } \\
\text { y determinar cómo encajan en una estructura } \\
\text { coherente. }\end{array}$ \\
\hline & $\begin{array}{l}\text { 8. Determinar un punto de vista, } \\
\text { valor, intención, o sesgo inherente a } \\
\text { un asunto en particular, con base en } \\
\text { las propias concepciones }\end{array}$ & $\begin{array}{l}\text { Demanda al profesor determinar un punto de vista, } \\
\text { realizar una valoración o inferir un sesgo inherente } \\
\text { a una situación planteada, con base en sus propias } \\
\text { concepciones. }\end{array}$ \\
\hline & $\begin{array}{l}\text { 9. Determinar un punto de vista, } \\
\text { valor, intención, o sesgo inherente a } \\
\text { un asunto en particular, con base en } \\
\text { un referente }\end{array}$ & $\begin{array}{l}\text { Demanda al profesor determinar un punto de vista, } \\
\text { realizar una valoración o inferir un sesgo inherente } \\
\text { a una situación planteada, con base en un recurso } \\
\text { informativo revisado previamente. }\end{array}$ \\
\hline Evaluar & $\begin{array}{l}\text { 10. Detectar logros y áreas de } \\
\text { oportunidad en el aprendizaje } \\
\text { logrado a través del curso } \\
\text { 11. Juzgar un producto }\end{array}$ & $\begin{array}{l}\text { Demanda al profesor realizar una autoevaluación } \\
\text { relacionada con su participación en el MOOC. } \\
\text { Demanda al profesor evaluar un producto } \\
\text { basándose en criterios o estándares establecidos } \\
\text { externamente. }\end{array}$ \\
\hline Crear & $\begin{array}{l}\text { 12. Generar acciones o alternativas } \\
\text { de solución para atender una } \\
\text { situación dada } \\
\text { 13. Diseñar un plan de trabajo para } \\
\text { conseguir un objetivo determinado } \\
\text { 14. Construir un producto original }\end{array}$ & $\begin{array}{l}\text { Demanda al profesor proponer acciones para } \\
\text { atender una situación problemática planteada. } \\
\text { Demanda al profesor idear un plan de acción para } \\
\text { alcanzar un objetivo. } \\
\text { Demanda al profesor sintetizar material } \\
\text { previamente revisado para la construcción de un } \\
\text { producto original. }\end{array}$ \\
\hline
\end{tabular}


Con la versión final de la TSA se efectuaron la segunda, tercera y cuarta ronda de codificación. A partir de la codificación se caracterizaron las actividades de aprendizaje clasificadas en cada segmento, lo cual se muestra en la Tabla 4.

\section{Tabla 4}

\section{Caracterización de las actividades clasificadas con la TSA}

\begin{tabular}{ll}
\hline Tipo de segmento & \multicolumn{1}{c}{ Características de las actividades de los MOOC AC } \\
\hline 1. Reconocer & Las actividades clasificadas en este tipo de segmento demandan identificar \\
información de la & respuestas correctas, con base en información previamente revisada de AC \\
memoria sobre un & durante la lección. Las actividades que pertenecen a este tipo de segmento pueden \\
tema revisado & consistir en: \\
& 1) Identificar si la premisa es verdadera o falsa. \\
& 2) Responder cuestionarios de opción múltiple. \\
3) Relacionar la información de dos columnas de manera correcta.
\end{tabular}

\section{Recuperar} información de la memoria sobre la experiencia personal

\section{Ejemplificar} acciones empleadas en la enseñanza

\section{Abstraer} información de un material o recurso revisado

\section{Comparar ideas}

6. Llevar a cabo una serie de indicaciones para elaborar una planeación

\section{Organizar conceptos o ideas}

\section{La actividad clasificada en este tipo de segmento se centra en recuperar} información sobre aspectos de la vida del profesor que no están relacionados con su trabajo en el aula. La única actividad clasificada en este rubro demanda al profesor recordar su propio proceso de alfabetización inicial.

Las actividades clasificadas en este tipo de segmento demandan al profesor explicar las acciones que implementa o ha implementado con los alumnos para favorecer su aprendizaje.

Las actividades clasificadas en este tipo de segmentos demandan al profesor recuperar información de un recurso, el cual se indica de manera explícita que debe ser revisado. Para recuperar la información de los recursos se proponen acciones como:

1) Identificar información específica.

2) Elaborar notas con información que el profesor considere relevante.

3) Completar información en tablas y esquemas preestablecidos.

Las instrucciones en actividades no requieren del docente emitir opiniones o juicios; en general, se orienta a resumir la información presentada.

Las actividades clasificadas en este tipo de segmento demandan al profesor comparar dos tipos de información, con el propósito de identificar diferencias y similitudes. Para esto se proponen acciones como:

1) Comparar el producto realizado con la respuesta correcta.

2) Identificar semejanzas y diferencias entre elementos del Programa de estudio 2011 y del Programa 2017.

3) Realizar comparación entre los productos derivados de las actividades del curso.

4) Identificar las diferencias y/o similitudes entre elementos del programa de AC, por ejemplo, identificar las diferencias entre los propósitos generales y los propósitos específicos.

Las actividades clasificadas en este tipo de segmento demandan la elaboración de una planeación, en cuyas instrucciones se indican una serie de aspectos que el profesor debe atender. Las planeaciones que deben elaborarse corresponden al trabajo de las asignaturas del campo de Formación Académica del programa de AC.

Las actividades clasificadas en este tipo de segmento demandan al profesor organizar información recuperada del programa de AC, la cual debe ser organizada en un esquema u organizador gráfico. 


\begin{tabular}{|c|c|}
\hline Tipo de segmento & Características de las actividades de los MOOC AC \\
\hline $\begin{array}{l}\text { 8. Determinar un } \\
\text { punto de vista, valor, } \\
\text { intención, o sesgo } \\
\text { inherente a un } \\
\text { asunto en particular, } \\
\text { con base en las } \\
\text { propias } \\
\text { concepciones }\end{array}$ & $\begin{array}{l}\text { Las actividades clasificadas en este tipo de segmento demandan al profesor emitir } \\
\text { una opinión sobre un tema en particular. Las opiniones o puntos de vista } \\
\text { solicitados se relacionan con la concepción del profesor sobre el aprendizaje de } \\
\text { diversas asignaturas, su opinión en situaciones hipotéticas del aula, o la } \\
\text { valoración de elementos del programa de AC. }\end{array}$ \\
\hline $\begin{array}{l}\text { 9. Determinar un } \\
\text { punto de vista, valor, } \\
\text { intención, o sesgo }\end{array}$ & $\begin{array}{l}\text { Las actividades clasificadas en este tipo de segmento demandan al profesor emitir } \\
\text { una opinión con base en la información revisada en el curso. La opinión que se le } \\
\text { solicita al docente puede ser en relación con: }\end{array}$ \\
\hline $\begin{array}{l}\text { inherente a un } \\
\text { asunto en particular, } \\
\text { con base en un } \\
\text { referente. }\end{array}$ & $\begin{array}{l}\text { 1) Los retos en la práctica a partir de lo que establece el material consultado. } \\
\text { 2) La valoración del contenido del programa de AC (en términos de utilidad o } \\
\text { importancia) para el trabajo en el aula. } \\
\text { 3) Argumentación en la elección de una respuesta. }\end{array}$ \\
\hline
\end{tabular}

10. Detectar logros y áreas de oportunidad en el aprendizaje logrado a través del curso

Las actividades clasificadas en este tipo de segmento demandan al profesor identificar los logros obtenidos a través de su experiencia en el curso, o bien, los aspectos que requiere fortalecer. Los logros se refieren a los aprendizajes conseguidos, así como a las experiencias exitosas de la puesta en práctica de lo revisado en el curso. Por otro lado, los aspectos a fortalecer se refieren a las áreas de oportunidad identificadas al poner en práctica lo revisado en el curso y al nivel de dominio de los contenidos estudiados.

11. Juzgar un Las actividades clasificadas en este tipo de segmento demandan al profesor producto evaluar, a partir de una lista de criterios, una actividad realizada en el curso. Las actividades consisten en emplear listas de cotejo para la evaluación de esquemas y planeaciones.

12. Generar acciones Las actividades clasificadas en este tipo de segmento demandan al profesor o alternativas de solución para proponer acciones para atender una situación dada. Las actividades están orientadas a proponer acciones para:

atender una

1) Favorecer el aprendizaje de los alumnos.

situación dada

2) Afrontar retos que se identifican en la práctica docente.

3) La gestión escolar.

4) Lograr que la educación sea inclusiva.

13. Diseñar un plan de trabajo para

Las actividades clasificadas en este tipo de segmento demandan al profesor elaborar un plan de acción con un objetivo específico, lo cual pueden consistir en: conseguir un objetivo determinado

1) Planear secuencias didácticas de diversas asignaturas.

2) Diseñar propuestas didácticas en una de las Áreas de Desarrollo Personal y

Social

3) Diseñar planes de acción orientados a fortalecer aspectos de la práctica docente.

14. Construir un producto original

Las actividades clasificadas en este tipo de segmento demandan al profesor elaborar un producto, material o recurso a partir de la síntesis de diferentes fuentes de información. Cabe señalar que en la mayoría de las actividades se fomenta la colaboración con otros profesores. En este tipo de segmento se proponen actividades como:

1) Redacción de ensayos.

2) Elaboración de carteles.

3) Elaboración de presentaciones.

4) Elaboración de esquemas a partir de la síntesis de otros esquemas.

A través de la codificación realizada con la TSA se identificó que existían similitudes en las actividades de aprendizaje propuestas, independientemente del grado o la temática del curso. Las tareas fueron similares en términos del nivel del proceso cognitivo y el tipo de productos a elaborar. Es así como se aprecia en el diseño del curso 
una reutilización de las actividades de aprendizaje, lo cual se da entre lecciones, así como en los distintos MOOC que conforman a la Colección de AC.

\section{Niveles taxonómicos del proceso cognitivo de las actividades de aprendizaje}

Enseguida se muestran los resultados del análisis de contenido cuantitativo en relación con la dimensión del proceso cognitivo asociado a las actividades de aprendizaje de la estructura general de la Colección de AC, es decir, de las 20 lecciones identificadas como únicas y diferentes y de los 152 segmentos de actividad. En los resultados se aprecian diferencias en la distribución de los procesos cognitivos. Cabe precisar que un criterio para identificar el proceso cognitivo del segmento de actividad fue valorarlo con el mayor nivel identificado, por ejemplo, una actividad clasificada en el nivel de Crear podría comprender procesos de niveles inferiores, como Recordar, Comprender o Aplicar. Con esto en consideración, en la Figura 3 se puede observar que una parte considerable de las actividades alcanzaron el nivel de Comprender, segundo nivel de la Taxonomía, así como de Analizar y Crear, cuarto y sexto nivel respectivamente.

\section{Figura 3}

Porcentaje de las actividades de aprendizaje según el nivel taxonómico del proceso cognitivo, con relación a la estructura general de los MOOC de la Colección de AC

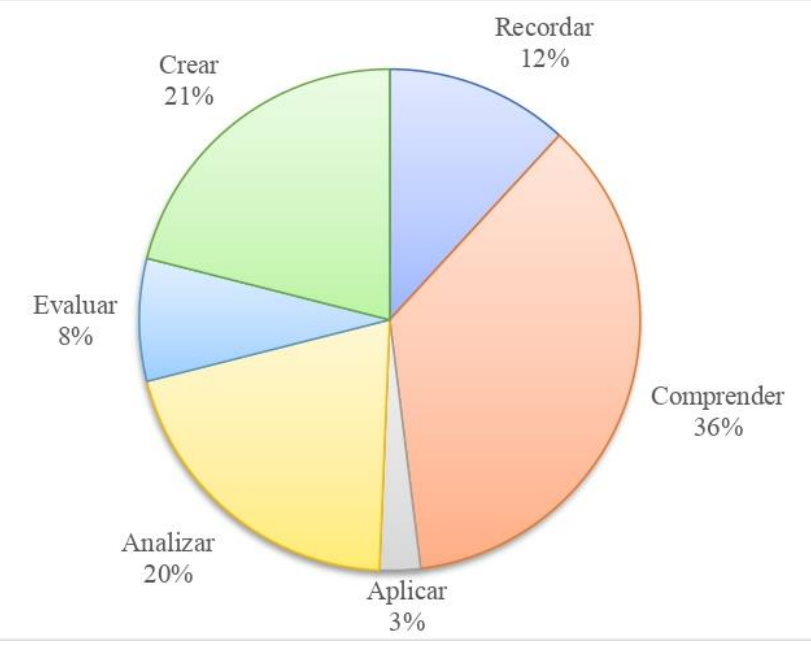

Asimismo, se obtuvieron resultados de la estructura general de la Colección de AC con relación a los TSA. En la Tabla 5 se muestra la frecuencia de las actividades de aprendizaje por nivel taxonómico. De los 152 segmentos identificados, los TSA con mayor representación son el 4 "Abstraer información de un material o recurso revisado" y 5 "Comparar ideas", ambos pertenecientes a la dimensión Comprender, seguidos por el segmento 12 "Generar acciones o alternativas de solución para atender una situación dada", de la dimensión Crear. Mientras que los segmentos con menor representación son el 2 "Recuperar información de la memoria sobre la experiencia personal", de la dimensión Recordar; el segmento 6 "Llevar a cabo una serie de indicaciones para elaborar una planeación", de la dimensión Aplicar; y el tipo 13 "Diseñar un plan de trabajo para conseguir un objetivo determinado", de la dimensión Crear. 


\section{Tabla 5}

Frecuencia y porcentaje de los Tipos de Segmento de Actividad de la estructura general del MOOC de Aprendizajes Claves

\begin{tabular}{|c|c|c|c|}
\hline Dimensión & Tipo de Segmento de Actividad (TSA) & Frecuencia & Porcentaje \\
\hline & 1. Reconocer información de la memoria sobre un tema revisado & 17 & 11.2 \\
\hline Recordar & $\begin{array}{l}\text { 2. Recuperar información de la memoria sobre la experiencia } \\
\text { personal }\end{array}$ & 1 & .07 \\
\hline \multirow{3}{*}{ Comprender } & 3. Ejemplificar acciones empleadas en la enseñanza & 5 & 3.3 \\
\hline & 4. Abstraer información de un material o recurso revisado & 28 & 18.4 \\
\hline & 5. Comparar ideas & 22 & 14.5 \\
\hline Aplicar & $\begin{array}{l}\text { 6. Llevar a cabo una serie de indicaciones para elaborar una } \\
\text { planeación }^{1}\end{array}$ & 4 & 2.6 \\
\hline \multirow{3}{*}{ Analizar } & 7. Organizar conceptos o ideas & 5 & 3.3 \\
\hline & $\begin{array}{l}\text { 8. Determinar un punto de vista, valor, intención, o sesgo } \\
\text { inherente a un asunto en particular, con base en las propias } \\
\text { concepciones }\end{array}$ & 9 & 5.9 \\
\hline & $\begin{array}{l}\text { 9. Determinar un punto de vista, valor, intención, o sesgo } \\
\text { inherente a un asunto en particular, con base en un referente }\end{array}$ & 17 & 11.2 \\
\hline \multirow{2}{*}{ Evaluar } & $\begin{array}{l}\text { 10. Detectar logros y áreas de oportunidad en el aprendizaje } \\
\text { logrado a través del curso }\end{array}$ & 5 & 3.3 \\
\hline & 11. Juzgar un producto & 7 & 4.6 \\
\hline \multirow{4}{*}{ Crear } & $\begin{array}{l}\text { 12. Generar acciones o alternativas de solución para atender una } \\
\text { situación dada }\end{array}$ & 20 & 13.2 \\
\hline & $\begin{array}{l}\text { 13. Diseñar un plan de trabajo para conseguir un objetivo } \\
\text { determinado }\end{array}$ & 4 & 2.6 \\
\hline & 14. Construir un producto original & 8 & 5.3 \\
\hline & Total & 152 & 100.0 \\
\hline
\end{tabular}

Con los resultados también se hizo una distinción de los procesos cognitivos de las actividades por lección de la estructura general de la Colección de AC. En la Figura 4 se observa que las lecciones no poseen una distribución uniforme en relación con los procesos cognitivos. En 9 de las 20 lecciones predominan actividades de aprendizaje ubicadas en la dimensión Comprender; en tres lecciones la mayor concentración recae en actividades ubicadas en la dimensión Crear; en tres lecciones predominan actividades de la dimensión Analizar; en una lección dominan actividades de la dimensión Evaluar; $\mathrm{y}$ en cuatro lecciones no hay predominancia de un tipo de segmento.

\footnotetext{
${ }^{1}$ En el comité de expertos se decidió incluir el TSA 6 dentro del nivel de aplicación, pues las actividades a las cuales aludía incluyen una serie de indicaciones que brindan al docente una guía detallada para elaborar una planeación. Mientras que en los TSA 12 y 13 sólo se plantea la problemática, sin dar orientaciones precisas de cómo resolverlo, por lo cual se considera que este tipo de actividades se asocian al proceso cognitivo de creación.
} 


\section{Figura 4}

Porcentaje de las actividades de aprendizaje según nivel taxonómico del proceso cognitivo, por lección de la estructura general del MOOC de Aprendizajes Claves

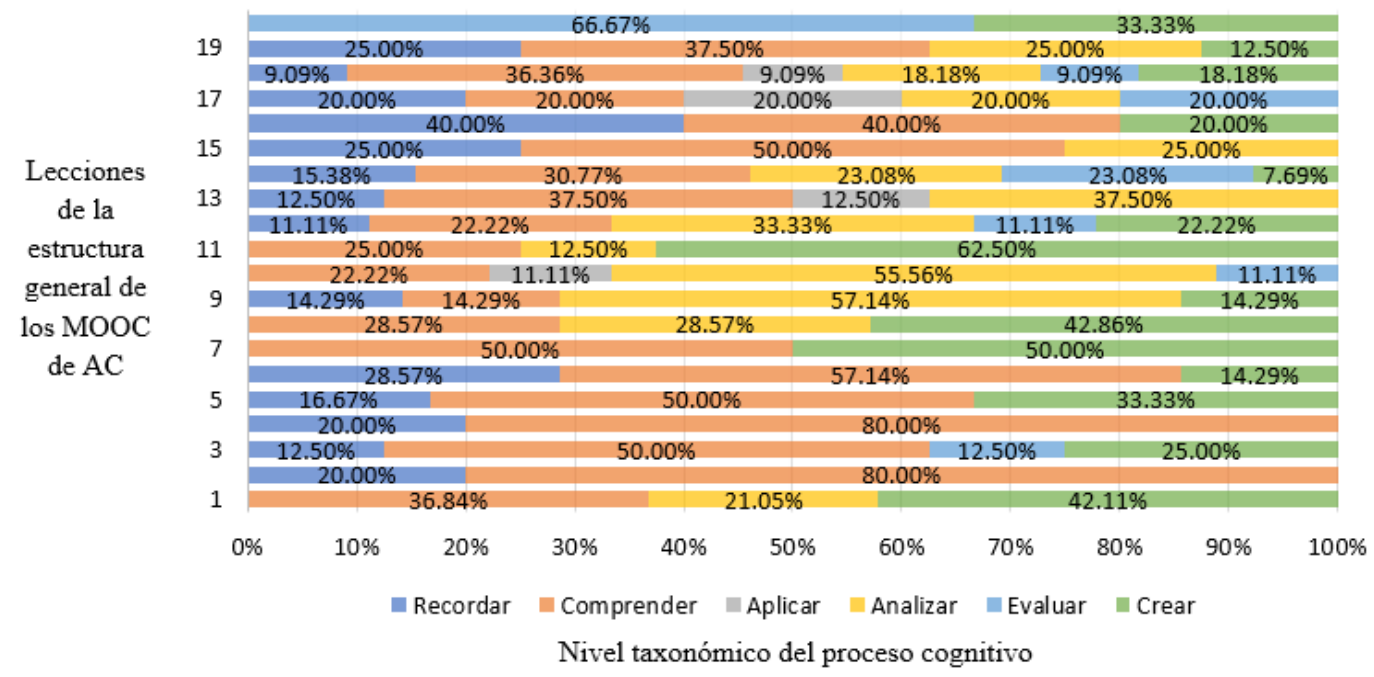

En la Figura 4 también se observó que no todas las lecciones comprenden los seis niveles taxonómicos del proceso cognitivo. Por ejemplo, en algunas lecciones las actividades se ubicaron en los niveles inferiores, como son el caso de la lección 2 y 4 , referentes a la dosificación curricular del programa de estudios, en las que sólo se identificaron actividades del Recordar y Comprender. Mientras que en otras se identificó una mayor orientación hacia los niveles superiores, como la lección 20 centrada en el cierre del curso y su evaluación, donde la atención recayó en los niveles de Evaluar y Crear. Asimismo, se identificó que 14 de las 20 lecciones incluyeron actividades de aprendizaje ubicadas en el nivel de Crear, el más alto considerado en la Taxonomía de Anderson y Krathwohl (2001), lo cual podría tener implicaciones favorables para la transferencia del aprendizaje, como señala Mayer (2002).

Con base en esto, se considera que cuatro de los TSA corresponden a actividades auténticas y posiblemente favorezcan la transferencia del aprendizaje, al aludir a actividades situadas en contextos reales en los que se ven implicados procesos cognitivos de las categorías superiores. Los TSA identificados son: 6 "Llevar a cabo una serie de indicaciones para elaborar una planeación", 10 "Detectar logros y áreas de oportunidad en el aprendizaje logrado a través del curso", 12 "Generar acciones o alternativas de solución para atender una situación dada" y 13 "Diseñar un plan de trabajo para conseguir un objetivo determinado".

De igual forma, se identificó cierta regularidad en la proporción del tipo de proceso en todos los grados, así como diferencias en las relaciones entre las lecciones de la estructura general. 


\section{Discusión y conclusiones}

En la Colección de AC el contenido temático está centrado en el conocimiento del Plan y los Programas de estudio para educación básica 2017, las actividades y productos de aprendizaje se encuentran previamente definidos, así como la secuencia para realizar y concluir el curso. La Colección de AC se identificó como un xMOOC, pues posee características propias de este tipo de cursos, como ser estructurado y se delimita claramente a partir del contenido (Yousef et al., 2014); se centra en la distribución del contenido, más que en la conformación de redes o en el conocimiento socialmente construido (Sobrino, 2014), además de proponer un aprendizaje lineal y guiado (Cabero et al., 2014).

La Colección de AC tiene un comportamiento ciertamente único. Respecto a las tasas de registro, se reporta que en la Colección de AC se inscribieron entre 900,000 y 1,000,000 de personas (DGFC, 2019; SEP, 2018b). Este dato supera por mucho el registro usual en plataformas como Coursera, que cuenta con un promedio de 11,000 matriculados por curso (Laurillard, 2016). Asimismo, la colección tuvo una tasa de finalización también muy alta, ya que es notoriamente superior a lo encontrado en la literatura. En el campo general de los MOOC se registra una tasa de éxito del $10 \%$ (Haber, 2014), mientras que en la Colección de AC registró una tasa del 50\% en el año 2018, según datos de la propia SEP (DGFC, 2019). De acuerdo con los datos de los 29,300 participantes que contestaron la encuesta de satisfacción aplicada al finalizar el MOOC, el curso fue valorado de manera satisfactoria, en relación con su estructura y organización, la relevancia del contenido y la claridad de los propósitos formulados para el curso. La mayoría de los participantes señaló también que el curso fue de utilidad en la mejora de su competencia pedagógica (SEP, 2018b).

Esta colección de MOOC es una opción formativa que tiene un nivel de acceso nunca visto en la historia de la formación y actualización de profesores en México. No obstante, como señala García-Aretio (2017), los MOOC no son la panacea en términos educativos y no son adecuados para todos los estudiantes, por lo cual es necesario valorar sus alcances.

Uno de los hallazgos de esta investigación fue la identificación de la reutilización de lecciones y actividades de aprendizaje en la estructura general de los seis MOOC que componen la Colección de AC. Como señalan Bartolomé y Steffens (2015), la creación y modificación de un curso completo implica una gran inversión, que en ocasiones no es redituable para las ofertas educativas en línea, por lo tanto, resulta conveniente la creación de unidades de aprendizaje, semejantes a los bloques de Lego, que pueden ser combinadas de diferentes formas y reemplazadas para la creación de una variedad de cursos, lo cual implicaría una menor inversión. Fue así como el conjunto de seis cursos se estructuró a partir de una batería de 20 lecciones, compuestas en total por 80 actividades de aprendizaje, sin contar que algunas de ellas solicitaban la realización de varios ejercicios y productos, además de que existía poca o nula articulación entre las mismas. Ante esta situación, se considera que la visión fragmentada y saturada de actividades del diseño de la formación podría ser un obstáculo para que los profesores lleguen a una generalización constructiva de lo aprendido que facilite la transferencia del aprendizaje en su aula.

Otro hallazgo que se identificó en la Colección de AC fue que se presentan las actividades de aprendizaje de manera no estructurada. Quintana y Tan (2019) señalan 
que los cursos estructurados deberían proveer una carga de trabajo similar en todas las unidades de aprendizaje, en términos de cantidad, secuencia y patrones, lo cual indica al alumno qué esperar en lecciones futuras; en cambio, un curso no estructurado se reconoce por una carga irregular de trabajo y una distribución desequilibrada del tiempo, así como la falta de información para orientar la realización de actividades. En los MOOC en cuestión se pudo notar que algunas lecciones incluían hasta 12 actividades, por ejemplo, la "Lección 1. Aprendizajes Clave para la educación integral. Plan y programa de estudio" en la cual se solicita la elaboración de al menos nueve productos escritos, la participación en un foro de discusión y dos actividades colaborativas. Mientras que otras, como la "Lección 3. Lengua Materna. Español: propósitos, enfoque pedagógico y evolución curricular", se propone la realización de sólo dos actividades, que consisten en la elaboración de dos productos escritos. Con base en lo anterior, se cree que la falta de organización y estructura en el diseño de las actividades podría representar una dificultad para los participantes, pues no tendrían una orientación clara sobre qué esperar al momento de avanzar en la realización de las tareas, además de dificultar la organización al momento de distribuir el tiempo estimado para realizar las lecciones del curso.

Por otra parte, se reconoció que los MOOC de la Colección de AC se orientaron mayormente a la comprensión del contenido curricular de Aprendizajes Clave. Derivado de la aplicación de la TSA se identificó que alrededor del $36 \%$ de las actividades se relacionan con la comprensión y el $20 \%$ con el análisis de la información. Se considera que es poco probable que un diseño basado mayormente en la comprensión del contenido sea la estrategia más adecuada para promover la transferencia del aprendizaje (Guskey, 2002), por lo que se requiere orientarlo hacia procesos cognitivos superiores, como son aplicación, análisis, evaluación y creación.

También se identificó que alrededor del $20 \%$ de los segmentos de actividad de aprendizaje que conforman los MOOC de la Colección de AC ofrecen oportunidades particulares para que los conocimientos logrados sean llevados a la práctica docente en el aula. Estas fueron reconocidas como actividades auténticas, pues se ajustan a la definición de Jonassen (1991, como se citó en Herrington et al., 2006) al corresponder a actividades que proporcionan niveles apropiados de complejidad y que poseen relevancia y utilidad en el mundo real. Para Herrington et al. (2006) estas tareas son la clave para una educación a distancia exitosa, las cuales corresponden a los niveles taxonómicos del proceso cognitivo de Crear, Aplicar y Evaluar. Por su parte, IbarraSáiz y Rodríguez-Gómez (2020) señalan que las tareas que implican la utilización de un pensamiento de alto nivel favorecen la transferencia del aprendizaje. Una actividad de esta naturaleza puede ser realizada por un período considerable de tiempo, e incluir en su interior una serie de actividades articuladas entre sí para conseguir un fin. Por ello se recomienda que, en lugar de basarse en un diseño con una gran variedad de actividades orientadas a la comprensión de un contenido, la formación se centre en una reducida cantidad de tareas bien estructuradas que conduzcan a los participantes a repensar el contenido en términos de la realidad de su aula y estén enfocadas a los procesos superiores para conseguir un aprendizaje significativo que pueda ser empleado y reinterpretado en una variedad de situaciones.

Se sabe que una condición para lograr la transferencia del aprendizaje es que los MOOC sean planificados intencionalmente con dicho propósito (Rivera et al., 2016). Es por ello que la construcción de un MOOC implica desafíos metodológicos que van más 
allá del componente tecnológico (González et al., 2016). Asimismo, si se tiene el foco de atención en la transferencia, es decir, la manera en que los docentes actualizan sus prácticas en la aplicación del nuevo plan de estudios no basta con verificar si con la formación se ha logrado algún aprendizaje, sino qué tipo de aprendizajes y por medio de qué procesos cognitivos se ha conseguido.

Se concluye que un MOOC que tiene el propósito de actualizar la práctica de docentes en servicio tendría que estar conformados por un alto porcentaje de actividades auténticas, que correspondan a los niveles taxonómicos superiores del proceso cognitivo y que sean de utilidad para la práctica profesional. Es así como, en el caso de la Colección de AC, pareciera que no es suficiente que el $20 \%$ de las actividades correspondieran a este tipo, por lo cual la recomendación es que se aumente este porcentaje para favorecer la formación profesional.

La TSA diseñada es un recurso útil para los creadores de cursos, pues permite tener claridad respecto a si las actividades se alinean a los objetivos y los aprendizajes propuestos, lo cual es de especial importancia en el campo de los MOOC dado que el diseño de la formación es el único medio de comunicación entre los formadores y el usuario debido al carácter autogestionado que los distingue de otros dispositivos de formación. La tipología también es un instrumento para realizar futuras evaluaciones de los alcances de los MOOC como herramienta de formación, ya que permite permite valorar las demandas cognitivas de un curso masivo y abierto como un elemento más para dimensionar los posibles alcances de sus objetivos.

Presentación del artículo: 26 de abril de 2021

Fecha de aprobación: 23 de junio de 2021

Fecha de publicación: 30 de septiembre de 2021

León, R., Cordero, G. y Luna, E. (2021). Estudio y evaluación del diseño de las actividades de aprendizaje de los MOOC de la Colección de Aprendizajes Clave. RED. Revista Educación a Distancia, 21(67). http://dx.doi.org/10.6018/red.478311

\section{Financiación}

Este estudio se ha realizado con el apoyo de la convocatoria de Ciencia Básica de CONACyT México (Proyecto no. A1-S-29890).

\section{Referencias}

Alemán, L., Sancho-Vinuesa, T. y Gómez, M. (2015). Los MOOC: ¿una transformación radical o una moda pasajera? Indicadores de calidad pedagógica para el diseño de un curso en línea masivo y abierto de actualización docente. International Journal of Educational Technology in Higher Education (ETHE), 12(1), 104-118. http://dx.doi.org/10.7238/rusc.v12i1.2260

Anderson, L.W. y Krathwohl, D. R. (Ed.). (2001). A Taxonomy for Learning, Teaching, and Assessing: A Revision of Bloom's Taxonomy of Educational Objectives. Longman. 
Badia, A. y Monereo C. (2004). La construcción de conocimiento profesional docente: análisis de un curso de formación sobre la enseñanza estratégica. Anuario de psicología, 35(1), 0047-70. https://ddd.uab.cat/record/71712

Bartolomé, A. y Steffens, K. (2015). ¿Son los MOOC una alternativa de aprendizaje? Comunicar, 22(44), 91-99. http://dx.doi.org/10.3916/C44-2015-10

Bozkurt, A., Keskin, Ö. N. y De Waard, I. (2016). Research Trends in Massive Open Online Course (MOOC) Theses and Dissertations: Surfing the Tsunami Wave. Open Praxis, 8(3), 203-221. http://dx.doi.org/10.5944/openpraxis.8.3.287

Cabero, J., Llorente, M. y Vázquez, A. (2014). Las tipologías de MOOC: su diseño e implicaciones educativas. Profesorado. Profesorado, Revista de Currículum y Formación de Profesorado, 18(1), 13-26.

http://www.ugr.es/ recfpro/rev181ART1.pdf

Castaño-Muñoz, J., Kalz, M., Kreijns, K. y Punie, Y. (2018). Who is taking MOOCs for teachers' professional development on the use of ICT? A cross-sectional study from Spain. Technology, Pedagogy and Education, 27(5), 607-624. https://doi.org/10.1080/1475939X.2018.1528997

Curtis, A. (2018). Online Professional Development. The TESOL Encyclopedia of English Language Teaching, 1-7.

Del Prete, A., y Cabero, J. (2020). El uso del Ambiente Virtual de Aprendizaje entre el profesorado de educación superior: un análisis de género. RED. Revista de Educación a Distancia, 62. http://dx.doi.org/10.6018/red.400061

Diario Oficial de la Federación. (2017). Acuerdo número 07/06/17 por el que se establece el Plan y los Programas de Estudio para la Educación Básica: Aprendizajes clave para la educación integral. (Continúa en la Tercera Sección). https://www.dof.gob.mx/nota_detalle_popup.php?codigo=5488475

Dirección General de Formación Continua, Actualización y Desarrollo Profesional de Maestros de Educación Básica. (2019). Aprendizajes Clave. Corte al 11 de diciembre de 2019.

Domínguez, J. y Morcillo, J. (2016). Evaluación de un curso en línea para la formación de competencias en el uso de las TIC en profesores de ciencias de secundarias públicas del sureste de México. RED. Revista de Educación a Distancia, (51), 1-25. https://revistas.um.es/red/article/view/275141

Duverger, M. (1983). Métodos de las ciencias sociales. Ariel.

Escobar-Pérez, J. y Cuervo-Martínez, A. (2008). Validez de contenido y juicio de expertos: una aproximación a su utilización. Avances en Medición, 6, 27-36. https://pdfs.semanticscholar.org/0736/455b135cfa8e5fc192d6bd526d1546b4528d.pd $\mathrm{f}$

García-Aretio, L. (2017). Los MOOC están muy vivos. Respuestas a algunas preguntas. RIED. Revista Iberoamericana de Educación a Distancia, 20(1). http://dx.doi.org/10.5944/ried.20.1.17488 
González, C.S., Collazos, C.A. y García, R. (2016). Desafío en el diseño de MOOCs: incorporación de aspectos para la colaboración y la gamificación. RED. Revista de Educación a Distancia, 48(7). https://revistas.um.es/red/article/view/253491

Guskey, T. R. (2002). Does it make a difference? Evaluating professional development. Educational, School, and Counseling Psychology Faculty Publications, 7. https://uknowledge.uky.edu/edp_facpub/7

Gynther, K. (2016). Design Framework for an Adaptive MOOC Enhanced by Blended Learning: Supplementary Training and Personalized Learning for Teacher Professional Development. Electronic Journal of e-Learning, 14(1), 15-30. http://www.ejel.org/issue/download.html?idArticle $=482$

Haber, J. (2014). MOOCs. The MIT Press.

Hayes, A. F. y Krippendorff, K. (2007). Answering the Call for a Standard Reliability Measure for Coding Data. Communication Methods and Measures, 1(1), 77-89. https://doi.org/10.1080/19312450709336664

Herrington, J., Reeves, T. y Oliver, R. (2006). Authentic tasks online: A synergy among learner, task, and technology. Distance Education, 27(2), 233-247.

https://doi.org/10.1080/01587910600789639

Ibarra-Sáiz, M.S. y Rodríguez-Gómez, G. (2020). Evaluando la evaluación. Validación mediante PLS-SEM de la escala ATAE para el análisis de las tareas de evaluación. RELIEVE, 26(1). http://doi.org/10.7203/relieve.26.1.17403

Instituto Nacional para la Evaluación de la Educación. (2015). Los docentes en México. Informe 2015. https://historico.mejoredu.gob.mx/publicaciones/los-docentes-enmexico-informe-2015/

Jornet, J. M., González-Such, J., Suárez, J. M. y Perales, M. J. (2011). Diseño de procesos de evaluación de competencias: consideraciones acerca de los Estándares en el dominio de las competencias. Revista Bordón, 63(1), 125-145.

King, M., Luan, B. y Lopes, E. (2018). Experiences of Timorese language teachers in a blended Massive Open Online Course (MOOC) for Continuing Professional Development (CPD). Open Praxis, 10(3), 279-287.

https://www.openpraxis.org/ openprax/index.php/OpenPraxis/article/view/840

Kirkpatrick, J. D. y Kirkpatrick, W. K. (2016). Kirkpatrick's Four Levels of Training Evaluation. Association for Talent Development.

Krippendorff, K. (2004). Content analysis. An introduction to its methodology. Sage

Laurillard, D. (2016). The Educational Problem that MOOCs Could Solve: Professional Development for Teachers of Disadvantaged Students. Research in Learning Technology, 24(1), 1-24. https://doi.org/10.3402/rlt.v24.29369

López-Aranguren, E. (2000). El análisis de contenido tradicional. En M. García, J. Ibañez y F. Alvira (Comps.), El análisis de la realidad social: métodos y técnicas de investigación (pp. 55-574). Alianza.

Estudio y evaluación del diseño de las actividades de aprendizaje de los MOOC de la Colección de Aprendizajes Clave. R. León, G. Cordero y E. Luna. 
Mabuan, R. A. (2018). Confessions of a Moocer: An Autoethnographic Inquiry on Online Distance Education. Turkish Online Journal of Distance Education, 19 (4), 198-213. https://doi.org/10.17718/tojde.471916

Maina, M. (2020). E-actividades para un aprendizaje activo. En A. Sangrá (Coord.), Decálogo para la mejora de la docencia online. Propuestas para educar en contextos presenciales Discontinuos (87-98). UOC.

Mayer, R. (2002). Rote Versus Meaningful Learning. Theory into Practice, 41(4), 226232. $\quad$ http://cmapspublic2.ihmc.us/rid=1Q2PTM7HL-26LTFBX9YN8/Krathwohl\%202002.pdf

Moreno, T. (21 de marzo de 2018). SEP: $56 \%$ de maestros se capacitaron para nuevo modelo educativo. El Universal. https://www.eluniversal.com.mx/estados/sep-56-demaestros-se-capacitaron-para-nuevo-modelo-educativo

Napier, A., Huttner-Loan, E. y Reich, J. (2020). Evaluating Learning Transfer from MOOCs to Workplaces: A Case Study from Teacher Education and Launching Innovation in Schools. RIED. Revista Iberoamericana de Educación a Distancia, 23(2), pp. 45-64. http://dx.doi.org/10.5944/ried.23.2.26377

Oyo, B., Kalema, B. M. y Byabazaire, J. (2017). Los MOOC para profesores en ejercicio: el caso de Uganda y las lecciones para África. Revista Española de Pedagogía, 75(266), 121-141. https://doi.org/10.22550/REP75-1-2017-07

Quintana, R. M. y Tan, Y. (2019). Characterizing MOOC Pedagogies: Exploring Tools and Methods for Learning Designers and Researchers. Online Learning, 23(4), 6284. http://dx.doi.org/10.24059/olj.v23i4.2084

Rivera, N., Ramírez, M., Hernández, D. y Sandoval, A. (2016). Diseño y transferencia del aprendizaje en un curso masivo abierto a distancia. Innovaciones Educativas, $18(25)$, 21-37. https://revistas.uned.ac.cr/index.php/innovaciones/article/view/1648/1868

Ruiz-Palmero, J., López-Álvarez, D. y Sánchez-Rivas, E. (2021). Revisión de la producción científica sobre MOOC entre 2016 y 2019 a través de SCOPUS. PixelBit, Revista de Medios y Educación, (60), 95-107. https://doi.org/10.12795/pixelbit.77716

Secretaría de Educación Pública. (2018a). Convocatoria Nacional para el registro en la oferta de cursos en línea "Colección de Aprendizajes Clave" Educación Básica. https://www2.sepdf.gob.mx/principal/archivos-convocatorias-2018/2018-02-06convocatoria.pdf

Secretaría de Educación Pública. (2018b). Resultados de formación continua 2018. https://dgfc.basica.sep.gob.mx/publications/pub/158/RESULTADOS+DE+FORMA CI\%C3\%93N+CONTINUA

Sobrino, A. (2014). Aportaciones del conectivismo como modelo pedagógico postconstructivista. Propuesta Educativa, (42), 39-48.

http://propuestaeducativa.flacso.org.ar/wp-content/uploads/2019/12/42-dossiermorras.pdf 
Tee, T., Yunos, J., Mohamad, B., Othman, W. y Yee, M. (2010) The Evaluation of Thinking Skills based on Taxonomy of Anderson and Krathwohl. En The 3rd Regional Conference on Engineering and Research on Higher Education (RCEE \& RHEd 2010), 7-9 June 2010, Kuching, Sarawak. http://library.oum.edu.my/repository/688/

Vázquez-Cano, E., López-Meneses, E., Gómez-Galán, J. y Parra-González, M.E. (2021). Innovative university practices on the educational advantages and disadvantages of MOOC Environments. RED. Revista Educación a Distancia, 21(66). http://dx.doi.org/10.6018/red.422141

Wambugu, P. W. (2018). Massive open online courses (MOOCs) for professional teacher and teacher educator development: A case of TESSA MOOC in Kenya. Universal Journal of Educational Research,6 (6), 1153-1157. https://doi.org/10.13189/ujer.2018.060604

Yousef, A. M. F., Chatti, M. A., Schroeder, U., Wosnitza, M. y Jakobs, H. (2014). A Review of the State-of-the-Art. Acta de CSEDU, 9-20.

https://www.researchgate.net/publication/275823066_MOOCs_a_review_of_the_stat e-of-the-art

Zapata-Ros, M. (2015). El diseño instruccional de los MOOC y el de los nuevos cursos abiertos personalizados. Revista de Educación a Distancia, (45).

https://revistas.um.es/red/article/view/238661 